\title{
BRCA-Monet: a breast cancer specific drug treatment mode-of-action network for treatment effective prediction using large scale microarray database
}

\author{
Chifeng Ma ${ }^{1}$, Hung-l Harry Chen ${ }^{2}$, Mario Flores ${ }^{1}$, Yufei Huang ${ }^{1,3^{*}}$, Yidong Chen ${ }^{2,3^{*}}$
}

From The International Conference on Intelligent Biology and Medicine (ICIBM 2013)

Nashville, TN, USA. 11-13 August 2013

\begin{abstract}
Background: Connectivity map (cMap) is a recent developed dataset and algorithm for uncovering and understanding the treatment effect of small molecules on different cancer cell lines. It is widely used but there are still remaining challenges for accurate predictions.

Method: Here, we propose BRCA-MoNet, a network of drug mode of action (MoA) specific to breast cancer, which is constructed based on the cMap dataset. A drug signature selection algorithm fitting the characteristic of cMap data, a quality control scheme as well as a novel query algorithm based on BRCA-MoNet are developed for more effective prediction of drug effects.

Result: BRCA-MoNet was applied to three independent data sets obtained from the GEO database: Estrodial treated MCF7 cell line, BMS-754807 treated MCF7 cell line, and a breast cancer patient microarray dataset. In the first case, BRCA-MoNet could identify drug MoAs likely to share same and reverse treatment effect. In the second case, the result demonstrated the potential of BRCA-MoNet to reposition drugs and predict treatment effects for drugs not in cMap data. In the third case, a possible procedure of personalized drug selection is showcased.

Conclusions: The results clearly demonstrated that the proposed BRCA-MoNet approach can provide increased prediction power to cMap and thus will be useful for identification of new therapeutic candidates.

Website: The web based application is developed and can be access through the following link http:// compgenomics.utsa.edu/BRCAMoNet/
\end{abstract}

\section{Background}

The ultimate goal of personalized medicine is to design treatments that optimize the therapeutic benefits and minimize the potential risk of toxicity for individual patient. Current pharmacogenomics research is striving to guide compound development and drug selection for such purpose. This growing need for personalized treatment

\footnotetext{
* Correspondence: Yufei.Huang@utsa.edu; cheny8@uthscsa.edu 'Department of Electrical and Computer Engineering, the University of Texas at San Antonio, One UTSA Circle, San Antonio, Texas, USA

${ }^{2}$ Greehey Children Cancer Research Institute, the University of Texas Health

Science Center at San Antonio, San Antonio, Texas, USA

Full list of author information is available at the end of the article
}

has pushed the development of high-through technologies such as microarray and high throughput sequencing to the research forefront, where compound selection methods based on DNA or mRNA profiling have been developed to achieve highest benefit from therapeutic intervention but at the same time lowest risk of side effects [1-6]. In the meantime, these high-throughput profiling technologies could be applied to elucidate the mechanism of compound treatment in inducing or inhibiting gene expression regulation at different levels. In this study, the focus is on using gene expression profiling for drug screen and effective treatment prediction. 
Besides genome-wide association studies, the current gene expression based approaches are mainly based on the "signature gene set" concept, which has been perfected during the past 14 years of relentless efforts in gene expression profiles of cancer, cardiovascular disease, diabetes and other disease researches. [7-10]. The key differences of this "signature gene set" approach from traditional linkagebased genetics study lie in two aspects. First, the "signature gene set" approach can identify genomic variation, being it in SNP, DNA copy number alteration, or miss-regulation of gene expression. Second, it can predict the relevant biological pathways, protein-protein interaction networks, and gene ontology functional groups, thus identifying novel therapeutic targets/biomarkers for drug discovery, with the hope that their variations from patient to patient could explain large portion of dosage variation, resistance and efficacy of the drug [11]. As such, one could also hypothesize that the activities (the relative abundance and interactions) of these signature genes could be part of drug targets, or mode-of-action (MoA), as these genes can be used to explain tumor types and differences in chemotherapeutic response of patients. In other words, activities of signature genes could be used to predict the drug sensitivity. In addition, one may extend this hypothesis further such that this prediction of pharmacological levels in cell type could be extrapolated to other cell types. Applications of these hypotheses have been developed in many studies $[12,13]$. One of the most notable work is the connectivity map (cMap) project [12], where 4 human cell lines (MCF7/ssMCF7, HL60, PC3, and SKMEL5) were treated by 1,309 chemical compounds at different dosages, and their expression profiles were generated. A prediction algorithm based on gene set enrichment analysis (GSEA) [14] was also developed to rank compounds based on input signature genes obtained from tumor comparison. This project has been widely adapted and developed in the drug discovery area. Several treatment candidates have been discovered for cancer cell lines in the cMap project by directly applying the cMap approach [15-17]. With the idea of searching for 'inverse signature' to the phonotype of interest, this approach has been extended to predict treatment potentials of compounds not included in the cMap project [18-22]. In addition to the original cMap approach, multiple other methods have been developed based on cMap data for new drug repositioning approaches [23-28] or improving the performance of exist cMap [29-31].

Although cMap has been widely applied, problems remain to be resolved for reliable prediction. First, cMap does not differentiate cell lines in its prediction. Often times, the top ranked drugs were from cell lines different from the query cell line. However, our investigation (see Result) suggested that the drug effect is cell line dependent and the higher ranks of the drugs from other cell lines would be more of cell line effects as opposed to drug effects. As a result, considering drug samples from other cell lines introduces only noise to drug prediction. Second, the quality of the data samples in cMap is inconsistent. Some samples from the same drug treatment can behave considerably different from the rest. These samples will inevitably present erroneous predictions. Third, the query signature gene set in cMap is chosen to include the top up- and down- regulated genes. However, size of the gene set is determined quite ad hoc. As a result, one might miss the important signature genes by choosing a smaller gene set, or on the contrary, bring in unrelated genes that would only serve to degrade the prediction. As an example, we used the expression data for estradiol (E2) treated MCF7 cell line [32] as a query to cMap and genes corresponding to the highest 100 and lowest 100 fold changes were used as the query gene set. Naturally, we would expect that E2 ranked high in the predicted list of drugs. However, E2 was only ranked 828 among over 1,200 drugs. The reason for this low ranking is because the result is a summary of the rankings of all cell lines of E2 samples, which are mixed (ssMCF7: rank 12, HL60: rank 31, MCF7: rank 3091, PC3: rank 3508; details in Additional file 1; see also BRCA-MoNet Application Case 1). However, even if we focused on E2 for MCF7 cell line, its ranking is still low (3091). Close look at the detailed results revealed that, the ranking E2 treated MCF7 cell line was a summary of the results from 19 individual E2 treated MCF7 cell line and their enrichment scores did not agree with each other (Table 1), Among the 19 samples, only a few have high enrichment scores. It is very likely that the rest of samples do not have high quality and thus fail to catch the real E2 treatment effect. Another potential cause for this poor result is the ineffective choice of the signature genes. However, as a user, we do not have a better way to choose an effective gene set to achieve better prediction. These results underscore the need for quality control and systematic selections of signature genes. To address the above challenges, we proposed BRCAMoNet in this paper. BRCA-MoNet is advantageous in

Table 1 Detailed prediction result in MCF7 cell line for an E2 treated query sample by the cMap project.

\begin{tabular}{cccccccc}
\hline Rank & batch & Cmap name & dose & cell & score & up & down \\
\hline 6 & 513 & estradiol & $10 \mathrm{nM}$ & MCF7 & .901 & .397 & -.307 \\
18 & 725 & estradiol & $100 \mathrm{nM}$ & MCF7 & .800 & .244 & -.381 \\
39 & 506 & estradiol & $100 \mathrm{nM}$ & MCF7 & .742 & .267 & -.314 \\
48 & 725 & estradiol & $10 \mathrm{nM}$ & MCF7 & .717 & .338 & -.223 \\
119 & 757 & estradiol & $100 \mathrm{nM}$ & MCF7 & .629 & .367 & -.125 \\
489 & 1010 & estradiol & $10 \mathrm{nM}$ & MCF7 & .432 & .179 & -.159 \\
731 & 656 & estradiol & $10 \mathrm{nM}$ & MCF7 & 0 & .454 & .187 \\
738 & 767 & estradiol & $10 \mathrm{nM}$ & MCF7 & 0 & .447 & .217 \\
741 & 506 & estradiol & $10 \mathrm{nM}$ & MCF7 & 0 & .445 & .195 \\
745 & 765 & estradiol & $10 \mathrm{nM}$ & MCF7 & 0 & .441 & .144 \\
\hline
\end{tabular}


three aspects compared with cMap. First, it focuses only on breast cancer cell line. Although doing so ignores other cell line data in the cMap data, it nevertheless removes the cell-line dependent interference from the true drug effect. Second, a quality control procedure as well as new drug signature gene set selection algorithm are developed to remove the possible noise in cMap data and characterize drug's treatment effect in a more systematic manner. Third, we define a Mode of Action (MoA) as a group of compounds that share the similar differential gene expression signature. Since the drug expression signature is indicative of the degree of its sensitivity to a cell, a MoA drug group should possess similar therapeutic effect. The construction of MoA introduces extra prediction power. This is because drugs with similar treatment effect might be ranked low due to high noise in data if we treat prediction of each drug independently. In contrast, this high noise sample could be ranked high because the query agrees with its MoA. The MoA is also different from other existing defined compound groups such as those by their anatomical therapeutic compound (ATC) classification since MoA is defined by differential gene expression after treatment, even though some overlapping between various compound classifications might be expected. The relationship of different MoAs in terms of their therapeutic effect can be modeled and visualized by a BRCA-MoNet. BRCAMoNet presents a global view of drug effects at a genomic level. This network augments and improves the current understanding of compound MoA defined mainly from a physiology perspective, and underscores the relationship of different compounds. From a computational perspective, the MoAs and the quantified relationship between drugs in BRCA-MoNet provide a system-level model crucial for optimal drug screening: a new compound can be easily assigned to a MoA in the BRCA-MoNet such that compound's therapeutic effectiveness can be extrapolated or inferred accordingly.

\section{Result}

\section{Analysis results showed drug treatment effect is cell line dependent}

In the cMap data, each drug treatment profile includes several treated samples from different cell lines. Whether the effects of the same drug treatments differ for different cell lines need to be investigated before a drug MoA network can be constructed. To this end, samples of cMap data were first grouped based on compounds and the compounds with more than 30 samples were retained. Note that since the data have already been normalized and fold changed over the control sample in the same cell line, the cell line dependent bias should be eliminated; any differences in expression levels within the samples of the same compound are manifestation of differences in chemo-effectiveness due to differences in cell line, drug concentrations, or a combination of both. Hierarchical clustering was performed to the samples in each compound group to reveal potential differences in expression patterns within the same compound. Correlating the clustering results with cell line types and concentrations (Figure 1A) revealed that chemo-effectiveness depends mainly on cell lines and is independent of concentration when it is effective. This finding is significant because it suggested that network construction and drug predictions should be performed by considering cell lines separately. Knowing the effect of one drug for treating breast cancer does not provide information on its effectiveness in lung cancer; including samples from cells other than breast cancer cells introduce only noise to drug treatment network construction. As a result, removing samples from other cells mitigates the interference and consequently improves the accuracy and robustness of the prediction result. Since MCF7 breast cancer cell line cohort contains the largest number of samples (2911 compared with HL60 1229 and PC3 1741), and it contains more drug replicate samples than other cell lines, we focused in this work on developing a breast cancer specific MoA network.

\section{Drug signature gene set selection}

The goal of signature gene set selection is to identify a set of genes that have significant differential expression after the drug treatment. However, the use of the conventional differential analysis methods such as $t$-test is hampered by the lack of the biological replicates in the cMap data set. This limitation becomes even severer after the quality control. For the MCF7 cell line, among all 1251 drugs in cMap, only 32 drugs have more than 5 samples and 1055 drugs have $\leq 2$ samples. With such small sample size, any statistically based differential analysis becomes infeasible. To this end, we proposed two criteria based on which the signature gene set of drug was selected: first, the signature genes should have high fold-change expression, and second, the fold change levels of the signature genes should be consistently high among the replicate samples. Based on these two criteria, new signature gene set selection algorithm tailored for small samples were developed (see METHOD for details). For MCF7 cell line, among 1251 drugs, signature gene sets of different size were identified for 1108 drugs. No gene sets were produced for the rest 118 drugs because no genes in their samples were consistently differential expressed. There are also 25 drugs which have only 1 sample in MCF7 cell line. As the result, these 118 MCF7 cell line inconsistent drugs as well as the 25 single-sample drugs were removed. Figure 1.C shows the identified signature gene sets for three drugs: Estradiol, estrol and raloxifene. Estradiol (E2) and Estrol are two forms of estrogen, which plays an important role in human breast cancer. It is therefore nature to see that the signature gene sets of these two drugs share many genes that also have similar 


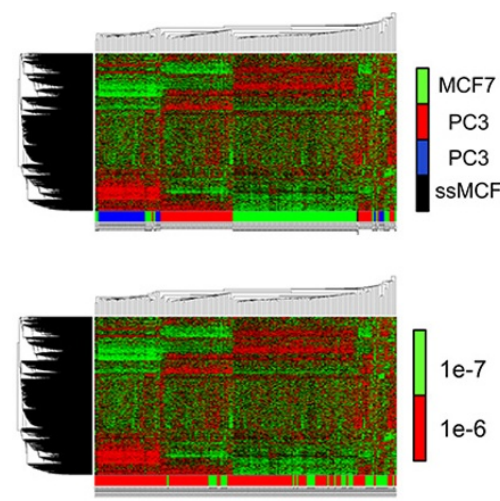

A

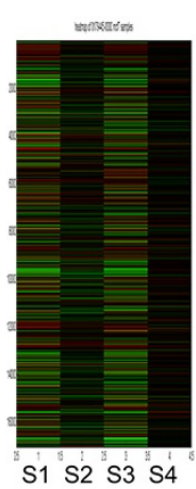

S1 S2 S3 S4
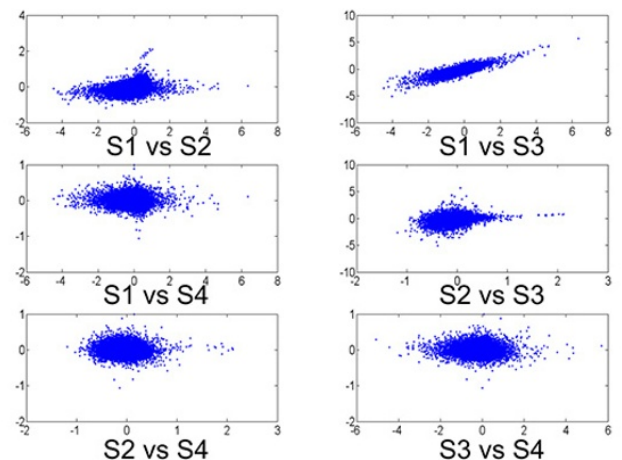

B
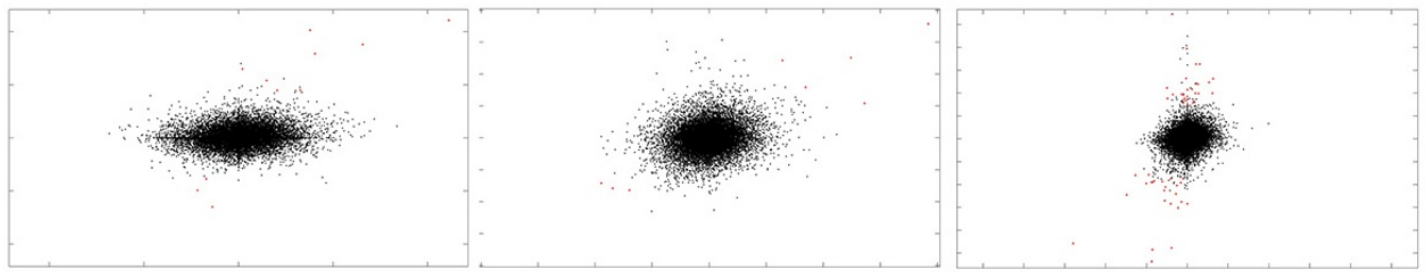

Estradiol

Estriol

Raloxifene

\begin{tabular}{|c|c|c|c|c|c|c|c|}
\hline Estradiol & & Estriol & & raloxifer & & & \\
\hline EGR3 & up regulate & EGR3 & up regulate & CXCL12 & down regulate & I17RB & down regulate \\
\hline MYBL1 & up regulate & MYBL1 & up regulate & GREB1 & down regulate & MYBL1 & down regulate \\
\hline C8orf44 // & up regulate & PIP5K1A & up regulate & EGR3 & down regulate & LPIN1 & up regulate \\
\hline ID1 & down regulate & FLNA & down regulate & CXCL12 & down regulate & LPIN1 & up regulate \\
\hline RET & up regulate & C9orf95 & up regulate & DDIT4 & up regulate & RASGRP1 & down regulate \\
\hline JAK2 & up regulate & EFNA1 & down regulate & CCNG2 & up regulate & PDZK1 & down regulate \\
\hline CXCL12 & up regulate & VAV3 & down regulate & & down regulate & INSIG1 & up regulate \\
\hline CXCL12 & up regulate & C8orf44 // & up regulate & STC2 & down regulate & SLC7A11 & up regulate \\
\hline CCNG2 & down regulate & & & GJA1 & down regulate & THBS1 & down regulate \\
\hline HSPA13 & up regulate & & & CCNG2 & up regulate & INSIGI & up regulate \\
\hline EFNA1 & down regulate & & & RET & down regulate & EPS15L1 & down regulate \\
\hline
\end{tabular}

Figure 1 Result of cell line investigation, signature selection, and quality control. A) The clustergram of expression samples from (HDAC) families of enzymes. Hierarchical clustering of 175 expression samples treated by drug TRS A. Rows and columns represent genes and samples, respectively. Columns were labeled with cell line (top) or concentrations (down). Clusters can be clearly observed and further examination of samples in the same cluster reveal that they are all from the same cell line. However, no such correspondence presented for the drug concentrations. This suggested that drug effectiveness is cell line dependent. B) Example of Quality Control. The heatmap and pair-wise twosample scatter-plot of 4 cMap samples from the same drug were shown. They revealed that only two samples showed similarly and the other two did not. In this example, sample s2 and s4 were removed as noise. C) Example of Signature Gene Set Selection. Two-sample scatter-plots of the selected gene signatures for three drugs were plotted. The red cross dots represented the selected genes and the black dots represented the rest of gene. Tables contain the symbols and expression up- or down- regulation for the selected genes of the three drugs.

expression patterns. For instance, genes EGR3, MYBL1 and C8orf33 are significantly up regulated and EFNA1 are down regulated after treated by both drug. Furthermore, these genes are highly relevant to breast cancer. EGR3 encodes a transcriptional regulator that belongs to EGR family and has been shown to be involved in the estrogen signaling pathway in breast cancer [33]. MYBL1 belongs to a group of genes that encode the MYB proto-oncogene protein; MYB has been shown to be highly expressed in ER + breast tumors and tumor cell lines and is essential for the proliferation of ER+ breast cancer cells [34]. EFNA1 encodes a member of the ephrin (EPH) family. It is highly compartmentalized in normal breast tissue and lost in invasive cancers; it is plausible to observe its down regulation 
after the E2 treatment. For the third drug, raloxifene, it is a known estrogen receptor modulator aiming at inducing the estrogen level. Our resulted signature includes both EGR3 and MYBL1 genes being down regulated. This similarity between the identified Estrol and Estradiol signature gene sets suggest that they may share similar MoA. In contrast, the reverse correlation between the raloxifene and E2 gene signatures suggest that their MoA may be opposite to each other. Later analysis indeed showed that E2 and Estrol as well as other 15 drugs are detected to be within the same MoA while roloxifene was predicted top ranked in the reverse prediction list with an independent E2 treatment sample (Details in BRCA-MoNet Application Case 1). These results demonstrated that the signature gene sets selected by our proposed algorithm are biologically meaningful.

\section{Quality control}

Quality control is applied on the drugs of cMap MCF7 cell line drugs with more than 3 samples. The goal of quality control is to remove the samples that are not consistently expressed with the others. Our investigation of the cMap data revealed that, there was a considerable amount of outlier samples, whose expression patterns differ significantly from the rest in the same drug (Figure 1B). Including these outliers would introduce only noise in defining the MoA and it is therefore important to remove the outlier samples. Note that signature gene set selection could also serve the purpose of quality control since some drugs could end selected no gene set. For MCF7 cell line, as the result of both gene signature selection and quality control, 1564 samples from 747 drugs are identified and removed and 1347 samples from 504 drugs are passed to BRACMoNet construction. These samples can be considered to correctly capture the treatment effect on the MCF7 cell line and were therefore used for subsequent investigation.

\section{Mode-of-Action \& BRCA-MoNet generation}

A compound mode of Action (MoA) is defined as a group of compounds that share similar gene signature expression patterns. Drugs forming one MoA will therefore have substantially shared genes in their signature gene set, which also have similar expression patterns. To obtain MoAs, clustering is applied to group the drugs with similar signature gene expression patterns. Multiple clustering algorithms exist and the simple yet effective Hierarchical Clustering $(\mathrm{HC})$ method is adopted in our work. There are two major reasons to choose HC. First, the number of clusters is not required for $\mathrm{HC}$; second, it is reasonable to expect that some drugs form distinct MoAs by itself and $\mathrm{HC}$ can produce clusters with a single member. To perform $\mathrm{HC}$, a distance matrix that measures pair-wise distances between drugs was obtained after quality control. With this distance matrix, a total of 109 MoAs were obtained at a threshold and a BRCA-MoNet (Figure 2) was constructed (see Method for details). In this network, each node represented one drug; a group of nodes share the same color edges represent a BRCA-MoA obtained by HC. For each MoA, the betweenness centrality of each drug was calculated and the drug with the largest betweenness
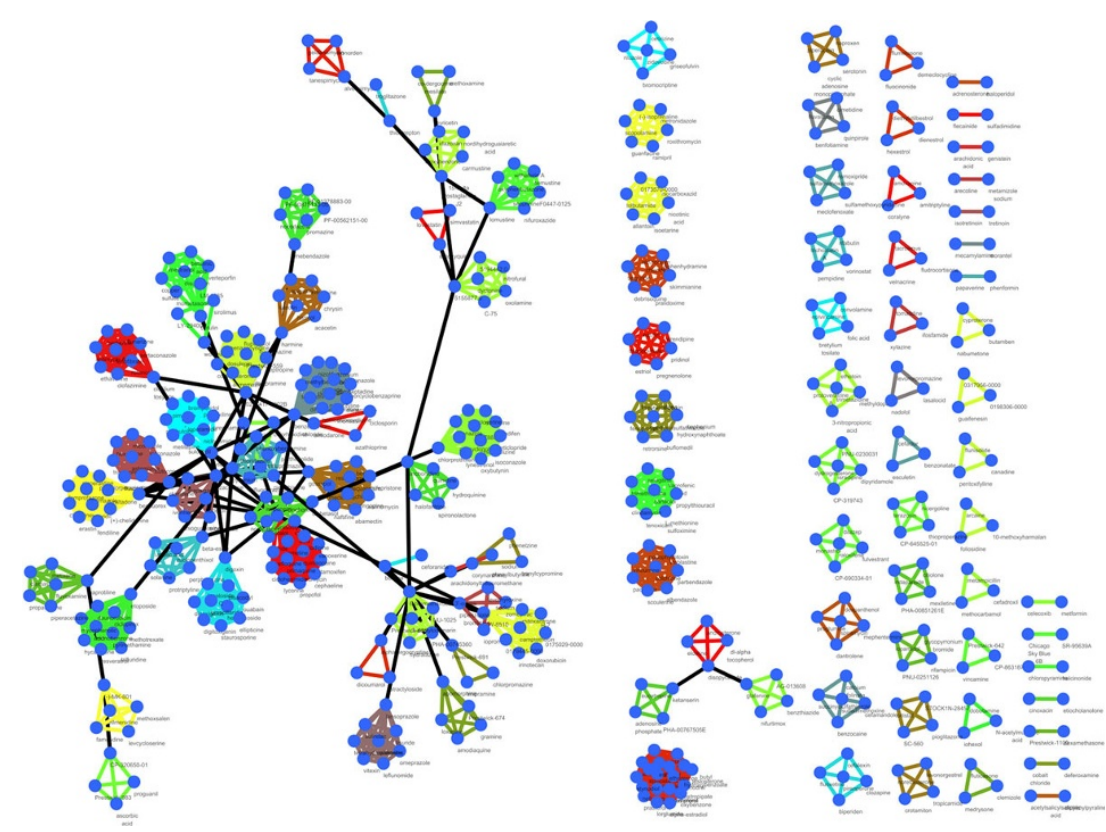

Figure 2 BRCA-MoNet. Each node represents a drug. A group of nodes linked by edges of the same color represent a MoA. The black edge linked two MoAs that show correlated effects. 
centrality was set to be the center of the MoA. Two MoAs were linked with a black edge if the distance between them was smaller than the threshold and this link indicated the secondary level relationship between two MoAs.

\section{BRCA-MoNet application}

After the BRCA-MoNet being constructed, its prediction power was tested. Three questions were investigated. First, can BRCA-MoNet predict correct drug MoA? Second, to what extent can BRCA-MoNet predict the drug MoA of an unknown or new drug? Third, to what extend can BRCA-MoNet recommend drugs for patients? To answer these questions, independent microarray expression datasets were downloaded from Gene Expression Omnibus (GEO) for the investigation. In order to avoid possible platform and experimental bias, the following criteria were followed when we select the data sets. First, the data must be compound treated on the MCF7 cell line and contain one or multiple control samples; this was consistent with the condition of the cMap data. Second, we only choose those datasets that were treated with drugs existed in the cMap project or of known treatment effect in breast cancer. Third, to avoid possible across platform complication, the data must be generated from the same platform as the cMap data, or GPL96 (Affymetrix Human Genome U133A Array). With the above considerations, the following three case studies were carried out.

\section{BRCA-MoNet application case 1: MoA prediction of E2 treated MCF7 cell line \& comparison with cMap}

We first chose the data set GSE 4025[35] as our query dataset. GSE 4025 includes the MCF7 cell line samples treated with 17beta-estrodiol (E2), a form of estrogen, for 24 hours. We pretended that we do not know the identity of compound (E2) and the goal was to use this treatment sample as a query to our BRCA-MoNet to predict its MoA. Note that E2 is a compound tested in the cMap data and also included in our BRCA-MoNet. Therefore, an accurate prediction algorithm would be expected to rank E2 associated MoA on the top of the predicted MoA list for similar treatment effect and possibly rank MoAs associated with estrogen receptor antagonist at the top of the reverse prediction list. The top similar predictions are shown in Table 2 (See Additional file 2 for the complete result). All the drugs are ranked based on their MoA gene signatures reversely related with $\mathrm{E} 2$. In the prediction result, the MoA (BRCA_MoA64) that contains E2 was ranked the 2nd among all the 109 MoAs and E2 is ranked the 4th among the total 504 MCF7 effective drugs selected for BRCAMoNet. This result indicates that our BRCA-MoNet can achieve very high precision. We investigated more closely the E2 associated BRCA_MoA64 and found that among 17 drugs, 11 are known to be related to estrogen. Specifically, three of them (Estropipate, alpha-estradiol, estrone) were
Table 2 Top 20 predicted drugs and corresponding BRC-MoA for similar effect prediction for E2.

\begin{tabular}{|c|c|c|c|}
\hline Rank & Drug & Mean Expression & MoA \\
\hline 1 & 0198306-0000 & 2.060663 & BRCA_MoA7 \\
\hline 2 & guaifenesin & 0.136039 & BRCA_MoA7 \\
\hline 3 & 0317956-0000 & 0.127664 & BRCA_MoA7 \\
\hline 4 & Estradiol & 2.002982 & BRCA_MoA64 \\
\hline 5 & norethisterone & 1.514845 & BRCA_MoA64 \\
\hline 6 & alpha-estradiol & 1.459319 & BRCA_MoA64 \\
\hline 7 & estropipate & 1.288005 & BRCA_MoA64 \\
\hline 8 & epitiostanol & 1.009552 & BRCA_MoA64 \\
\hline 9 & nefopam & 0.84901 & BRCA_MoA64 \\
\hline 10 & kaempferol & 0.795197 & BRCA_MoA64 \\
\hline 11 & noretynodrel & 0.76267 & BRCA_MoA64 \\
\hline 12 & levonorgestrel & 0.71241 & BRCA_MoA64 \\
\hline 13 & naringenin & 0.554154 & BRCA_MoA64 \\
\hline 14 & aminophylline & 0.541906 & BRCA_MoA64 \\
\hline 15 & etynodiol & 0.389381 & BRCA_MoA64 \\
\hline 16 & ethisterone & 0.239267 & BRCA_MoA64 \\
\hline 17 & epiandrosterone & 0.207091 & BRCA_MoA64 \\
\hline 18 & oxybenzone & 0.176137 & BRCA_MoA64 \\
\hline 19 & megestrol & -0.40088 & BRCA_MoA64 \\
\hline 20 & estrone & -0.81855 & BRCA_MoA64 \\
\hline
\end{tabular}

E2 is ranked $4^{\text {th }}$ (highlighted).

different forms of estrogen and six others (Norethisterone, ethisterone, norethynodrel, levonorgestrel, etynodiol, megestrol) are different forms of progestogen, a precursor of estrogen. Epiandrosterone can induce androgenic activity, which can also lead to a precursor of estrogen, and Epitiostanol is a form of anti-estrogen. Among the remaining six drugs, Naringenin is a weak estrogenic bioflavonoid that exhibits anti-estrogenic activity [32]; Aminophylline is known to interact with estrogen [36]; kaempferol is a dietary flavonoid that functions as a selective estrogen receptor modulator [37-39]; Oxybenzone (also known as benzophenone-3) is a compound widely used in the sunscreen and a few studies suggested that oxybenzone mimics the effects of the estrogen and may cause higher risk to breast cancer; Lorglumide has been shown to induce opposite effect of estrogen in [40]; only nefopam has no evidence that suggests any interaction with estrogen and breast cancer. This significant over-representation of the estrogen related compounds in the E2 associate MoA provides strong evidence to suggest that the constructed MoAs in our BRCA-MoNet do contain drugs of similar effect. Next, we predicted the MoAs with the reverse treatment effect. The result (Table 3; Additional file 3) is equally promising. In the highest ranked MoA (BRCA-MoA 80), two of three drugs (raloxifene, fulvestrant) are selective estrogen receptor modulators, which have anti-estrogenic actions, and the other one (monastrol) is an anti-breast cancer drug [41]. The second ranked MoA, BRCA-MoA86, contains one 
Table 3 Top 20 drugs and corresponding BRC-MoA for reverse effect prediction for E2

\begin{tabular}{cccl}
\hline Rank & Drug & Mean Expression & MoA \\
\hline 1 & monastrol & -3.54584 & BRCA_MoA80 \\
2 & fulvestrant & -1.64228 & BRCA_MoA80 \\
3 & raloxifene & -1.08114 & BRCA_MoA80 \\
4 & bacampicillin & -0.39068 & BRCA_MoA86 \\
5 & cyproterone & -0.95575 & BRCA_MoA52 \\
6 & nabumetone & -0.32439 & BRCA_MoA52 \\
7 & proguanil & -0.32213 & BRCA_MoA102 \\
8 & deferoxamine & -2.16577 & BRCA_MoA50 \\
9 & cobalt chloride & -0.25959 & BRCA_MoA50 \\
10 & wortmannin & -0.60183 & BRCA_MoA32 \\
11 & LY-294002 & -0.5634 & BRCA_MoA32 \\
12 & sirolimus & -0.2227 & BRCA_MoA32 \\
13 & metampicillin & -0.65666 & BRCA_MoA10 \\
14 & cefadroxil & -0.25222 & BRCA_MoA10 \\
15 & methocarbamol & -0.19939 & BRCA_MoA10 \\
16 & N-acetylmuramic acid & -0.44691 & BRCA_MoA33 \\
17 & dobutamine & -0.3141 & BRCA_MoA33 \\
18 & iohexol & -0.15247 & BRCA_MoA33 \\
19 & adrenosterone & -0.94798 & BRCA_MoA58 \\
20 & butamben & -0.41198 & BRCA_MoA58 \\
\hline & & &
\end{tabular}

drug: bacampicilin. Bacampicilin is a penicillin antibiotic and study showed that it interacts with estrogen to reduce the effect of estrogen [42]. The third ranked MoA, BRCAMoA52, contains two drugs: cyproterone and nabumetone.
Cyproerone is a steroidal anti-androgen with additional pro-gestogen and anti-gonadotropic properties. It can suppress the activity of the androgen hormones and subsequently reduce the productivity of estrogen. It has also been studied in clinical I and II trail for its potential as an anti-breast-cancer drug [43].

This query data were also applied to the original cMap prediction, where the most up- and down-regulated 200 genes were used as the query signature genes. As expected, the cMap project gave a mix results (Table 4) in both predictions of similar-effect drugs (with positive enrichment score) and reverse-effect drugs (with negative enrichment score). E2 itself only ranked 828 (Table 5) in the total 1309 compounds. In cMap, the rank was a summary of a drug's prediction results in every sample of all different cell lines. E2 has a lot of samples in the cMap data across all 5 cell line and the enrichment scores of these samples have large variations, ranging from 0.707 (ssMCF7) to -0.040 (PC3) (Table 6), and this large variation led an insignificant prediction rank. In the reverse effect prediction, Raloxifene, anti-estrogenic modulator, was found to be at rank 9 (Table 4) as expected, but fulvestrant, another anti-estrogenic modulator, only ranked 861 (Table 7). A closer look at the detailed cell line results revealed that fulvestrant had a negative enrichment score in the MCF7 cell line but a positive enrichment score in the HL60 cell line and the combined result led to a low rank. (Table 8) Over all, the comparison between prediction results of cMap and

Table 4 Top 20 prediction result generated from cMap using GSE4025 E2 treatment signature as a query

\begin{tabular}{|c|c|c|c|c|c|c|}
\hline Rank & Cmap name & Mean & $\mathrm{N}$ & Enrichment & $\mathbf{P}$ & Specificity \\
\hline 1 & MS-275 & -0.954 & 2 & -1.000 & 0.00000 & 0.0217 \\
\hline 2 & Vorinostat & -0.697 & 12 & -0.855 & 0.00000 & 0.0266 \\
\hline 3 & Trichostatin A & -0.597 & 182 & -0.735 & 0.00000 & 0.0000 \\
\hline 4 & Cephaeline & -0.564 & 5 & -0.889 & 0.00006 & 0.0361 \\
\hline 5 & Scriptaid & -0.701 & 3 & -0.962 & 0.00018 & 0.0000 \\
\hline 6 & Prochlorperazine & -0.276 & 16 & -0.506 & 0.00018 & 0.0472 \\
\hline 7 & Mefloquine & -0.481 & 5 & -0.805 & 0.00064 & 0.0278 \\
\hline 8 & 5252917 & 0.657 & 2 & 0.977 & 0.00087 & 0.0000 \\
\hline 9 & Raloxifene & -0.465 & 7 & -0.670 & 0.00114 & 0.0130 \\
\hline 10 & perhexiline & -0.476 & 4 & -0.821 & 0.00193 & 0.0168 \\
\hline 11 & Ceforanide & 0.199 & 4 & 0.813 & 0.00235 & 0.0207 \\
\hline 12 & Bacitracin & 0.516 & 3 & 0.890 & 0.00252 & 0.0068 \\
\hline 13 & Ikarugamycin & -0.525 & 3 & -0.886 & 0.00292 & 0.0152 \\
\hline 14 & Iloprost & 0.353 & 3 & 0.876 & 0.00354 & 0.0197 \\
\hline 15 & Isoxican & 0.290 & 5 & 0.722 & 0.00375 & 0.0549 \\
\hline 16 & Diethylstilbestrol & 0.413 & 6 & 0.666 & 0.00399 & 0.0737 \\
\hline 17 & Bufexamac & -0.404 & 4 & -0.786 & 0.00426 & 0.0000 \\
\hline 18 & Norcyclobenzaprine & -0.404 & 4 & -0.778 & 0.00501 & 0.0310 \\
\hline 19 & Riboflavin & -0.412 & 4 & -0.777 & 0.00511 & 0.0000 \\
\hline 20 & arachidonyltrifluoromethane & 0.576 & 2 & 0.946 & 0.00535 & 0.0000 \\
\hline
\end{tabular}


Table 5 cMap overall prediction result for E2

\begin{tabular}{lllllll}
\hline rank & Cmap name & mean & $\mathbf{N}$ & Enrichment & $\mathbf{P}$ & Specificity \\
\hline 828 & Estradiol & 0.209 & 37 & 0.367 & Null & Null \\
\hline
\end{tabular}

BRCA-MoNet shows that BRCA-MoNet adds considerable prediction power to the existent cMap data and greatly improves the prediction accuracy on both similar and reverse prediction.

\section{BRCA-MoNet Application Case 2: Prediction of BMS- 754807 Treated MCF7 Cell Line}

One additional dataset treated with drug BMS-754807 was tested against our BRCA-MoNet. This dataset (GSE33366) came from breast xenograft MCF7 bearing mice treated with BMS-754807. MBS-784807 is a dual IGF-1R/InsR inhibitor that can synergize hormonal agents and has been shown to be a potential breast cancer drug [44-47]. Study showed that there is an elevated IGF-IR activity specific in triple negative breast cancer and because of that, BMS784807 could be a possible treatment for triple negative breast cancer [48]. It has been investigated in several Phase I and Phase II Clinical Trials as an anti-cancer drug [49-52]. This dataset was tested against our BRCA-MoNet for similar treatment effect predictions. The top ranked MoA was MoA 37 (Table 9 and Additional file 4 for complete prediction). Interestingly, this MoA contains valproic acid, which is ranked number 1 among all the 504 BRCAMoNet drugs. Valproic acid belongs to a general class of drugs called anticonvulsants and was originally used as a non-opioid pain reliever. It has also been used to prevent migraine headaches [53]. Recently, valproic acid has been shown to have great potential as an epigenetic drug for anti-cancer activity through inhibiting cancer cell proliferation in various types of cancer [54-56]. This prediction result shows that both drugs with great anti-cancer potential are actually detected to have similar MoA by BRCAMoNet. This conclusion strongly supports the fact that BRCA-MoNet can uncover new drug's anti-cancer MoA by assigning it to a known MoA.

BRCA-MoNet application case 3: prediction of drugs for UNC breast cancer patients

Prediction power of BRCA-MoNet on the real breast cancer patients was investigated. To this end, dataset GSE2740

Table 6 cMap detail prediction result for E2

\begin{tabular}{lllllll}
\hline Rank & $\begin{array}{l}\text { Name and cell } \\
\text { line }\end{array}$ & Mean & $\mathbf{N}$ & Enrichment & $\mathbf{P}$ & Specificity \\
\hline 12 & Estradiol-ssMCF7 & 0.700 & 2 & 0.989 & 0.00016 & 0.0000 \\
31 & Estradiol-HL60 & 0.303 & 8 & 0.590 & 0.00335 & 0.1585 \\
3091 & Estradiol-MCF7 & 0.222 & 19 & 0.485 & Null & Null \\
3580 & Estradiol-PC3 & -0.040 & 8 & 0.223 & Null & Null \\
\hline
\end{tabular}

Table 7 cMap overall prediction result for fulvestrant

\begin{tabular}{lllllll}
\hline Rank & $\begin{array}{l}\text { Cmap } \\
\text { name }\end{array}$ & Mean & $\mathbf{N}$ & Enrichment & $\mathbf{P}$ & specificity \\
\hline 861 & fulvestrant & -0.295 & 40 & -0.352 & Null & Null \\
\hline
\end{tabular}

[57] was downloaded from GEO. This dataset includes samples from 4 platforms (GPL885, GPL887, GPL1390, and GPL1708) and various breast cancer subtypes. To avoid possible bias due to platforms and breast cancer subtypes, only patient samples of Lumina A (LumA) subtype and from the platform with the largest sample size (GPL1390) were chosen. A total of 97 breast cancer patients' microarray data samples were tested against our BRCA-MoNet using the reverse prediction. The ranking result is shown in Figure 3-A (detailed in additional file 5). Particular, several BRCA-MoAs were consistently ranked at the top, where BRCA-MoA24 ranked the first in 30.21\% of the all the patients and ranked above top 20 in $61.46 \%$ of all the patients among all 109 BRCA-MoAs. BRCA-MoA24 includes five drugs: spironolactone, rifabutin, vorinostat, trichostatin A and CP-690334-01. Among these five drugs, spironolactone is a synthetic, steroidal anti-mineralocorticoid agent with anti-androgen, weak pro-gestogen properties, and indirect estrogen effects. It has been used to reduce the elevated or unwanted androgen activity in the body [58]. (Androgen, as mentioned before, is the precursor of all estrogens.) So, spironolactone can be potentially used to induce anti-estrogenic activity against breast cancer. Rifabutin is a semisynthetic ansamycin and primarily used in the treatment of tuberculosis. Interestingly, ansamycin has been found to be a HSP90 inhibitor and many of its synthetic compounds are on trials as anti-breast cancer drug. [59-61] Vorinostat is a member of a histone deacetylases (HADC) with a broad spectrum of epigenetic activities; it has been approved by the FDA to treat cutaneous T-cell lymphoma in 2006. Since it has been also shown to have effect on treating breast cancer [62-68], it has undergone multiple Phase I and II clinical trials as an anti breast cancer drug [69-73]. Trichostatin A (TSA) is an organic compound that serves as an antifungal antibiotic and selectively inhibits class I and II mammalian HADC families of enzymes [74]. It has gained extremely high attention in

Table 8 cMap detail prediction result for fulvestrant

\begin{tabular}{lllllll}
\hline Rank & $\begin{array}{l}\text { Name and } \\
\text { cell line }\end{array}$ & Mean & $\mathbf{N}$ & Enrichment & $\mathbf{P}$ & specificity \\
\hline 5 & $\begin{array}{l}\text { Fulvestrant- } \\
\text { MCF7 }\end{array}$ & -0.595 & 21 & -0.714 & 0.00000 & 0.0146 \\
180 & $\begin{array}{l}\text { Fulvestrant- } \\
\text { HL60 }\end{array}$ & 0.178 & 6 & 0.442 & 0.13728 & 0.2086 \\
2466 & $\begin{array}{l}\text { Fulvestrant- } \\
\text { ssMCF7 }\end{array}$ & 0.000 & 1 & -0.594 & Null & Null \\
3274 & Fulvestrant-PC3 & -0.032 & 12 & 0.413 & Null & Null \\
\hline
\end{tabular}


Table 9 Top 20 drugs for similar effect prediction of BMS-754807

\begin{tabular}{cccc}
\hline Rank & Drug & Mean Expression & MoA \\
\hline 1 & valproic acid & 0.159748 & BRC_MoA37 \\
2 & clobetasol & 0.142458 & BRC_MoA37 \\
3 & labetalol & 0.110684 & BRC_MoA37 \\
4 & tiapride & 0.104771 & BRC_MoA37 \\
5 & cinchonine & 0.017354 & BRC_MoA37 \\
6 & imipenem & -0.00274 & BRC_MoA37 \\
7 & norfloxacin & -0.09407 & BRC_MoA37 \\
8 & idazoxan & 0.158088 & BRC_MoA12 \\
9 & nordihydroguaiaretic acid & 0.080311 & BRC_MoA12 \\
10 & carmustine & 0.028793 & BRC_MoA12 \\
11 & 15 -delta prostaglandin J2 & 0.021515 & BRC_MoA12 \\
12 & 5155877 & -0.0044 & BRC_MoA12 \\
13 & 5194442 & -0.01294 & BRC_MoA12 \\
14 & dioxybenzone & -0.04312 & BRC_MoA12 \\
15 & C-75 & -0.04929 & BRC_MoA12 \\
16 & (+)-chelidonine & 0.15705 & BRC_MoA1 \\
17 & prochlorperazine & 0.032193 & BRC_MoA1 \\
18 & erastin & 0.002357 & BRC_MoA1 \\
19 & famprofazone & -0.02239 & BRC_MoA1 \\
20 & clotrimazole & -0.02361 & BRC_MoA1 \\
\hline
\end{tabular}

recent years and has been actively studied for its potent antitumor activity against breast cancer ever since 2001 [75-79]. Although the information of the last drug (CP690334-01) is not available, the overrepresentation of breast cancer related drugs in this MoA gives us a clear vision of the significant detection power of BRCA-MoNet when applied to real patient data.

\section{Conclusion}

A drug effect MoA network for breast cancer cell lines, BRCA-MoNet, was constructed by using the cMap expression data. It was developed to address the problems of the cMap algorithm and to provide robustness and more accurate predictions for treatment effectiveness prediction and drug screening. This improvement came partially as a result of careful quality control on cMap data. In contrast to cMap, BRCA-MoNet prediction is cell line specific and removes the burden for user to select an effect signature gene set. Moreover, BRCA-MoNet assesses the therapeutics influence based on MoA instead of those for individually drugs. This network model not only leads to improved prediction results but it also uncovered the underlying MoA structure of the cMap data that has not been fully discovered before.

The case studies we analyzed here returned favorable results and insightful leads. For the E2 treated MCF7 cell line case, the detection power and insight of the BRCAMoNet E2-related MoA were exploited. The BMS-754807 case showed that BRCA-MoNet is capable of assigning new anti-cancer drug to the existing anti-cancer MoA and yielding insight understanding of drug MoA detection. The UNC breast cancer patients' case demonstrated the potential of BRCA-MoNet to be used as a tool for personalized treatment recommendation based on patients' gene expression.

The BRCA-MoNet approach provides added values to the connectivity map project and allowed for new and better capability in identification of possible therapeutic candidates. Future direction will likely lend itself to two paths: to expand the MoNet concept to other cancer and cell lines by incorporating multiple drug treatment dataset, and to mature BRCA-MoNet's capability of prediction for the real patients. We expect that the rapid development in cancer profiling projects including The Cancer Genome Atlas (TCGA) will greatly benefit our effort in these future directions

\section{Method}

\section{BRCA-MoNet workflow}

The proposed scheme of generating a breast cancer specific MoA network or BRCA-MoNet from cMap data is

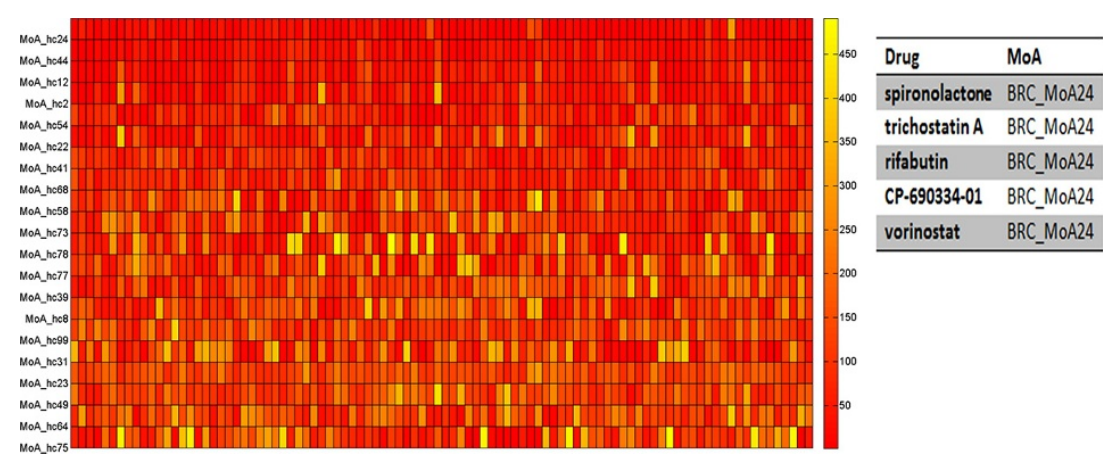

Figure 3 Prediction result of breast cancer patient. A) Top MoAs for reverse effect prediction for UNC lumA patients. Color of the heat map indicates the predicted rank of the MoA in an increasing order from red to yellow. B) Drugs of BRC-MoA24. 


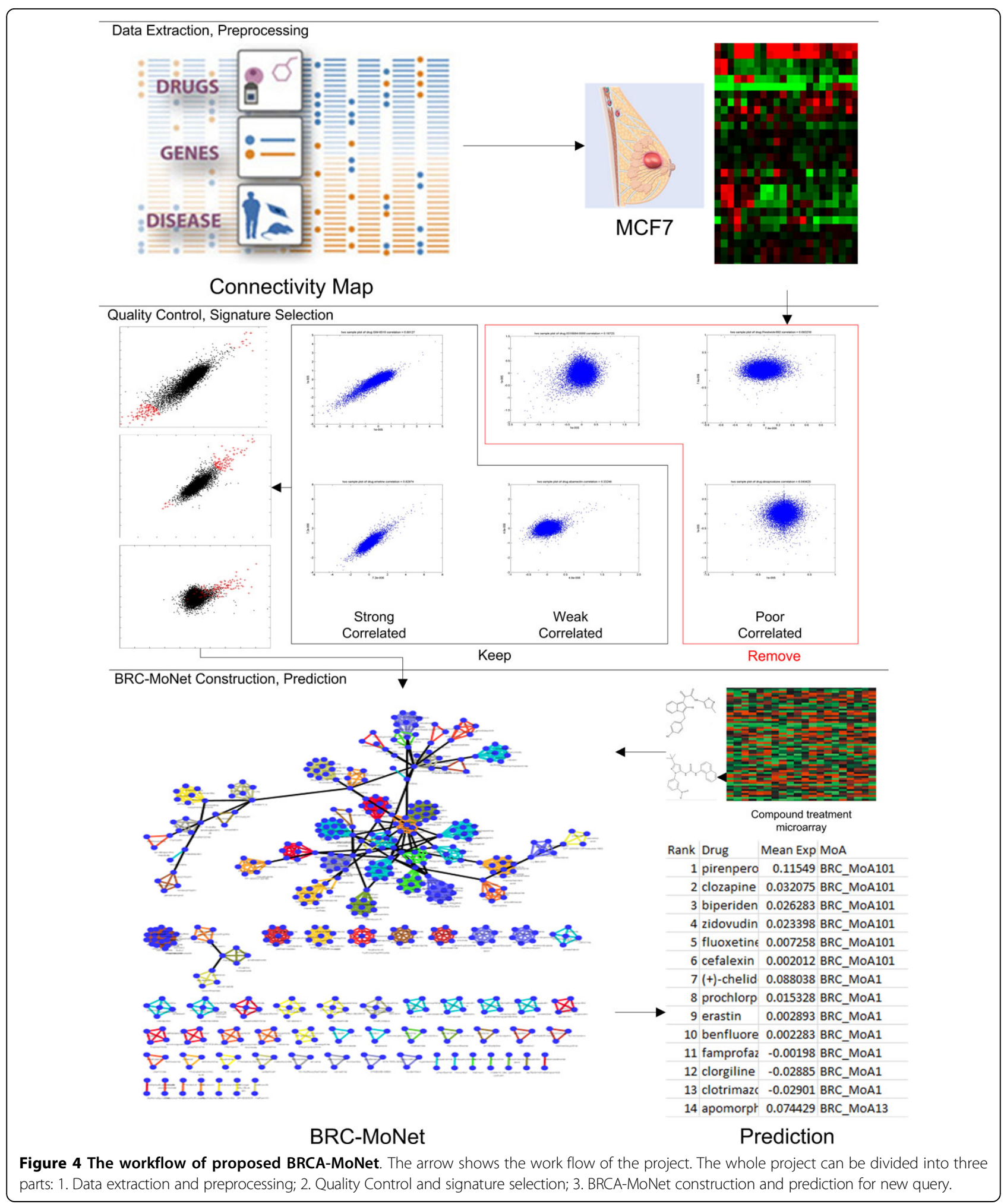

summarized in Figure 4. In the first step, new data preprocessing, drug signature selection and clustering algorithms were developed and applied to identify MoAs. In the second step, the relationship between the MoAs in terms of their effectiveness was assessed. Based on the MoAs, the BRCA-MoNet was constructed to depict the relationship of compound effectiveness. BRCA-MoNet and the drug signatures were used for subsequent prediction. 
Two types of prediction can be carried out with BRCAMoNet including similar prediction and reverse prediction. For the purpose of find the drug effectiveness on a tumor sample, the expression profile of an individual tumor sample is used as a query, where reverse prediction is adopted and the query will be inverse correlated against the MoAs to predict treatment effects. The prediction result includes a list of MoAs ranked in an increasing order of their negative correlation to the tumor profile. Since effective compounds are expected to have an adverse effect to tumor, MoAs with the negative correlations with the tumor profile will likely be candidates of choice for treating this individual tumor. For the purpose of finding a new compound's treatment effect, a query expression profile from treated sample of a new compound would be used instead as an input to BRCA-MoNet and both similar and reverse prediction results will be of interest as they are the compounds of respective similar and adverse effectiveness in expression. The BRCA-MoNet can be updated when new compound-treated expression profiles are available. One can take the advantage of existing BRCA-MoNet and update it by simply introducing a new MoA and their relationship to other groups. The algorithms are discussed in details in Methods.

\section{Data preparation}

Gene expression profiles of compound treatments were downloaded from Broad Institute's Connectivity Map web site (http://hRp://www.broadinsUtute.org/cmap/). Two Affymetrix arrays were utilized in this study (excluding 184 arrays from early-access version of HT-HG-U133A): HG-U133A (total of 807 arrays) and HT-HG-U133A (6029 arrays), representing 1,267 compound treatments at different dosages. In addition, data includes 5 cell lines: HL60, PC3, SKMEL5 and MCF7/ssMCF7. Each treated sample is accompanied by multiple control/vehicle samples. As for the normalization, the Perfect-Match(PM) probe level intensities, obtained from one Affymetrix array type (including treated and untreated hybridization), was first performed background adjustment together by using Robust Multi-array Average (RMA) procedure. after RMA background adjustment for both array types, quantile normalization was performed to all untreated samples; treated samples were then partitioned according to the array type, vehicle cell-line, and compound; for each group (same array type, cell-line and compound; rank-invariant normalization was performed against their corresponding untreated samples (base line of the normalization was the median of untreated vehicles) at probe-level to correct possible nonlinear abnormality. After normalization, the treated samples expression values were calculated by median polish procedure. At last, all samples (treated and untreated, and both array types) were reassembled into matrix according to Affymetrix probe set IDs.

\section{Signature gene set selection and distance assessment}

The goal of signature gene set selection is to select the genes that are expressed differentially. Since most of the drugs in cMap contains only two samples, the conventional differentially analysis algorithms such as t-test cannot be applied. We proposed the following test statistic to measure if a gene, say $i$, is consistently differentially expressed in a pair of samples

$$
R_{i}=\frac{x_{i}}{\sigma_{x}} * \frac{y_{i}}{\sigma_{y}}-\left|\frac{x_{i}}{\sigma_{x}}-\frac{y_{i}}{\sigma_{y}}\right|
$$

Where $x_{i}$ and $y_{i}$ is the expression of gene $i$ in sample $\times$ and sample y, respectively, and $\sigma_{x}$ and $\sigma_{y}$ are the corresponding sample standard deviation. This statistic values genes which are most differentially expressed in both samples, while taking the sample variation into the consideration. The empirical distribution of this statistic $R$ under the null hypothesis that the gene is not differentially expressed can be obtained by random sampling from replicates of the cMap data. Based on the distribution, p-values can be computed for every gene. A signature gene set of any paired drug samples are determined to contain gene with p-value $<0.1 \%$. The algorithm is summarized in Figure 5. For drugs having a larger sample sized than 2 , the procedure of determining signature gene set are fairly the same. Each pair of sample would be used to determine a gene set and then a common subset of all determined gene sets will be the final signature set. Based on the above selected signature gene sets, the distance $D_{a b}$ between any two drug treatment samples $a$ and $b$ is defined as

$$
D_{a b}=D_{\max }-\frac{1}{2} *\left(\frac{1}{n} \sum_{i^{n}} \frac{g_{(a-b) i}}{\operatorname{var}(b)}+\frac{1}{m} \sum_{j^{m}} \frac{g_{(b-a) j}}{\operatorname{var}(a)}\right)
$$

where $D_{\max }$ is the maximum distance among all pairwise drug treatment samples', $g_{(a-b) i}$ is the $i$ th gene expression level of sample a signature gene set in sample b,n and $m$ are the size of the signature gene sets (the total numbers of genes) for sample a and b, respectfully, and $\operatorname{var}(a)$, and $\operatorname{var}(b)$ are the sample variance of $a$ and $b$, respectfully.

\section{Quality control}

Quality control is done in two rounds of processing. In the first round, which is part of the gene selection, some drugs came by with no signature gene sets; this is a result that no genes were consistently differentially expressed in samples from this drug. The samples from those drugs were removed. Although some drugs were determined with a signature gene set, one or more of the outlier samples may not agree with the rest. To address this problem, a second round of further quality control process was also performed on the cMap samples. In order to remove these inconsistent samples, a new scheme was proposed in Figure 6. 


\section{Scheme of quality control}

For a drug A with treatment sample $n$ :

1 for each sample of the drug $A$ treatments $s_{A_{i}}$, calculate its pair wise distance $\left\{D_{A_{i-j}}: j=1 \ldots n, j \neq i\right\}$;

2 calculate the threshold $D_{t}$ from the empirical distribution of with-in drug pair wise distances;

3 remove the sample if $\max \left\{D_{A_{i-j}}\right\}<D_{t}$

Figure 5 Pseudo code of the proposed gene set selection scheme.

\section{MoA and MoNet generation}

According to the definition of MoA, two compounds are in the same MoA if they share the same genomic signature. This is equivalent to say that the samples from these two compounds are highly correlated. In contrast, the samples from different MoAs should have a correlation distributed according to the distribution of the population correlation. To determine if two drugs $i$ and $j$ belong to a MoA, a hypothesis testing formulation is developed with the null hypothesis defined by

$$
H_{0}: D_{i j} \sim p_{b}(D)
$$

where $D_{i j}$ is the Distance assessment between sample $i$ and $j$, and $p_{b}(D)$ is the the distribution of the population distance. $p_{b}(\rho)$ is estimated empirically based on the pairwise distances between all sample pairs of the same cell line. Then, a $p$ value of 0.01 is chosen as the significance level and the corresponding distance is determined as the threshold. Hierarchical clustering is performed on all the samples distances; then clusters are determined by cutting the linkage at the threshold and the resulted clusters were defined as the MoAs. Notice that since each MoA was generated totally based on the threshold obtained from the background distribution, some MoAs may contain large number of samples while other MoAs only contain few samples from one or two drugs; this is natural and reasonable because some compounds just do not share the treatment effectiveness with others.

Once the MoAs were identified, it was then desirable to reveal the relationship of the MoAs in terms of their therapeutic effects. Instead of investigating individual compound in an isolated fashion, MoNet will enable research to explore a set of compounds (MoAs) that share the same MoA-Signature genes (potential targets), as well as their correlated MoAs.

\section{Drug Effectiveness Prediction}

Using the MoNet and the MoA, one can 1) predict drug effectiveness of a new compound (Similar Prediction) and/or 2) screen compounds to predict the therapeutic

\section{Scheme of determining the signature gene set}

1 for each paired samples $x$ and $y$, determine the signature gene set

1) Calculates test statistics $R_{i}$ for each gene in the paired samples.

2) Calculate sorted list $R_{i_{-} \text {ord }}$ of all the $R_{i} s$ in a descend order.

3) Check the threshold $R_{t}$ from the empirical distribution with p-value $0.1 \%$.

4) Determine the signature gene set $G_{s_{-} i}$ so that every gene $g_{s}$ fulfill the criteria

$$
\left\{g_{s} \in G_{s_{-}}: R_{g_{s}} \leq R_{t}\right\}
$$

$$
2 \text { choose the gene set } G_{s-\max }=\max \left\{G_{s_{-} i} s\right\}
$$

Figure 6 Pseudo code of the proposed quality control scheme. 
effectiveness of different compounds if applied to an individual tumor (Reverse Prediction). For drug effectiveness prediction, the expression profile of cells/tissue treated by a new compound needs to be obtained and the goal is to identify the MoA of the compound. For the therapeutic prediction, a query gene expression profile of the tumor sample is required. The goal is to determine the degree of the adverse relationship between the MoAs and the tumor marker genes expression that reveals how likely the compound is to reverse the expression of tumor marker genes. From the perspective of algorithm development, prediction of drug effect and compound screening are essentially the same. The only difference is the distance criteria: When similar prediction is applied, the MoA is first ranked for the largest positive distance and then each drugs within the MoA are then ranked with the same criteria; when reverse prediction is applied, then the MoA is first ranked for the smallest negative distance and then each drugs within each MoA are ranked the same.

\section{Additional material}

Additional file 1: Detailed cMap prediction result for E2 treatment
query.
Additional file 2: Detailed BRCA-MoNet prediction result for similar
prediction of individual E2 treatment query.
Additional file 3: Detailed BRCA-MoNet prediction result for reverse
prediction of individual E2 treatment query.
Additional file 4: Detailed BRCA-MoNet prediction result for similar
prediction of individual BMS784807 treatment query.
Additional file 5: Detailed BRCA-MoNet prediction result for breast
cancer patient microarray dataset.

List of abbreviations used

Connectivity map: cMap; Mode of action: MoA; Breast Cancer Mode of Action Network: BRCA-MoNet; Hierarchical Clustering: HC; Gene Expression Omnibus: GEO; 17beta-estrodiol: E2; The Cancer Genome Atlas: TCGA.

\section{Competing interests}

The authors declare that they have no competing interests.

\section{Authors' contributions}

$\mathrm{CM}, \mathrm{YH}$, and $\mathrm{YC}$ conceived the idea and designed the experiments. $\mathrm{CM}$ and $\mathrm{HC}$ prepared the data and conducted the experiments. MF developed the web application. MF, YH and YC wrote the paper.

\section{Acknowledgements}

This work is supported by a National Science Foundation grant (CCF1246073) to YH and YC, a Qatar National Research Fund grant (09-874-3-235) to $\mathrm{YC}$ and $\mathrm{YH}$, and a National Institute of Health grant (NIH-NCATS UL1TR000149) to YC

\section{Declarations}

The publication fees were supported by Qatar National Research Fund grant (09-874-3-235)

This article has been published as part of BMC Systems Biology Volume 7 Supplement 5, 2013: Selected articles from the International Conference on Intelligent Biology and Medicine (ICIBM 2013): Systems Biology. The full contents of the supplement are available online at http://www. biomedcentral.com/bmcsystbiol/supplements/7/S5.

\section{Authors' details}

'Department of Electrical and Computer Engineering, the University of Texas at San Antonio, One UTSA Circle, San Antonio, Texas, USA. ${ }^{2}$ Greehey Children Cancer Research Institute, the University of Texas Health Science Center at San Antonio, San Antonio, Texas, USA. ${ }^{3}$ Department of Epidemiology and Biostatistics, University of Texas Health Science Center at San Antonio, San Antonio, Texas, USA.

Published: 9 December 2013

\section{References}

1. Sotiriou C, Pusztai L: Gene-expression signatures in breast cancer. N Engl J Med 2009, 360(8):790-800.

2. Riedel RF, et al: A genomic approach to identify molecular pathways associated with chemotherapy resistance. Mol Cancer Ther 2008, 7(10):3141-9.

3. Schlueter PJ, Peterson RT: Systematizing serendipity for cardiovascular drug discovery. Circulation 2009, 120(3):255-63.

4. Ebi $\mathrm{H}$, et al: Relationship of deregulated signaling converging onto mTOR with prognosis and classification of lung adenocarcinoma shown by two independent in silico analyses. Cancer Res 2009, 69(9):4027-35.

5. Hait WN, Hambley TW: Targeted cancer therapeutics. Cancer Res 2009, 69(4):1263-7, discussion 1267.

6. Garman KS, et al: A genomic approach to colon cancer risk stratification yields biologic insights into therapeutic opportunities. Proc Natl Acad Sci USA 2008, 105(49):19432-7.

7. Yamashita T, et al: EpCAM and alpha-fetoprotein expression defines novel prognostic subtypes of hepatocellular carcinoma. Cancer Res 2008, 68(5):1451-61.

8. Jia $\mathrm{HL}$, et al: Gene expression profiling reveals potential biomarkers of human hepatocellular carcinoma. Clin Cancer Res 2007, 13(4):1133-9.

9. Budhu $A$, et al: prediction of venous metastases, recurrence, and prognosis in hepatocellular carcinoma based on a unique immune response signature of the liver microenvironment. Cancer Cell 2006, 10(2):99-111.

10. Ye $\mathrm{QH}$, et al: predicting hepatitis B virus-positive metastatic hepatocellular carcinomas using gene expression profiling and supervised machine learning. Nat Med 2003, 9(4):416-23.

11. Kessel AGV: Large Scale Genome Variation in Health and Disease in: Cytogenetics and Genome Research.Nijmegen 2006.

12. Lamb J: The Connectivity Map: a new tool for biomedical research. Nat Rev Cancer 2007, 7(1):54-60.

13. Lee $\mathrm{JK}$, et al: A strategy for predicting the chemosensitivity of human cancers and its application to drug discovery. Proc Natl Acad Sci USA 2007, 104(32):13086-91

14. Subramanian A, et al: Gene set enrichment analysis: a knowledge-based approach for interpreting genome-wide expression profiles. Proc Natl Acad Sci USA 2005, 102(43):15545-50.

15. Huang $L$, et al: An integrated bioinformatics approach identifies elevated cyclin E2 expression and E2F activity as distinct features of tamoxifen resistant breast tumors. PLoS One 2011, 6(7):e22274.

16. Cohen $\mathrm{AL}$, et al: A pharmacogenomic method for individualized prediction of drug sensitivity. Mol Syst Biol 2011, 513.

17. Yeh $C T$, et al: Trifluoperazine an antipsychotic agent inhibits cancer stem cell growth and overcomes drug resistance of lung cancer. Am J Respir Crit Care Med 2012, 186(11):1180-8.

18. Yuen HF, et al: TAZ Expression as a Prognostic Indicator in Colorectal Cancer. PLoS One 2013, 8(1):e54211.

19. Edris $B$, et al: Comparative gene expression profiling of benign and malignant lesions reveals candidate therapeutic compounds for leiomyosarcoma. Sarcoma 2012, 805614.

20. Reka AK, et al: Identifying inhibitors of epithelial-mesenchymal transition by connectivity map-based systems approach. J Thorac Oncol 2011, 6(11):1784-92.

21. Claerhout $\mathrm{S}$, et al: Gene expression signature analysis identifies vorinostat as a candidate therapy for gastric cancer. PLoS One 2011, 6(9):e24662.

22. Lan MY, et al: From NPC therapeutic target identification to potential treatment strategy. Mol Cancer Ther 2010, 9(9):2511-23.

23. Lee $H S$, et al: Rational drug repositioning guided by an integrated pharmacological network of protein, disease and drug. BMC Syst Biol 2012, 80 . 
24. Gottlieb A, et al: pREDICT: a method for inferring novel drug indications with application to personalized medicine. Mol Syst Biol 2011, 496.

25. $\mathrm{Hu} \mathrm{G}$, Agarwal P: Human disease-drug network based on genomic expression profiles. PLoS One 2009, 4(8):e6536.

26. Shats I, et al: Using a stem cell-based signature to guide therapeutic selection in cancer. Cancer Res 2011, 71(5):1772-80.

27. Jin $\mathrm{G}$, et al: A novel method of transcriptional response analysis to facilitate drug repositioning for cancer therapy. Cancer Res 2012, 72(1):33-44.

28. Zhao $C$, et al: Identifying mechanistic similarities in drug responses. Bioinformatics 2012, 28(14):1902-10.

29. Shigemizu $D$, et al: Using functional signatures to identify repositioned drugs for breast, myelogenous leukemia and prostate cancer. PLOS Comput Biol 2012, 8(2):e1002347.

30. McArt DG, Zhang SD: Identification of candidate small-molecule therapeutics to cancer by gene-signature perturbation in connectivity mapping. PLoS One 2011, 6(1):e16382.

31. $\mathrm{Ma} \mathrm{H}$, Zhao H: FacPad: Bayesian sparse factor modeling for the inference of pathways responsive to drug treatment. Bioinformatics 2012, 28(20):2662-70.

32. Bulzomi $P$, et al: Naringenin and 17beta-estradiol coadministration prevents hormone-induced human cancer cell growth. IUBMB Life 2010, 62(1):51-60.

33. Inoue A, et al: Transcription factor EGR3 is involved in the estrogensignaling pathway in breast cancer cells. J Mol Endocrinol 2004, 32(3):649-61.

34. Drabsch Y, Robert RG, Gonda TJ: MYB suppresses differentiation and apoptosis of human breast cancer cells. Breast Cancer Res 2010, 12(4):R55.

35. Frasor J, et al: Gene expression preferentially regulated by tamoxifen in breast cancer cells and correlations with clinical outcome. Cancer Res 2006, 66(14):7334-40

36. Greenway FL, Bray GA, Heber D: Topical fat reduction. Obes Res 1995, 3(Suppl 4):561S-568S.

37. Guo AJ, et al: Kaempferol as a flavonoid induces osteoblastic differentiation via estrogen receptor signaling. Chin Med 2012, 10

38. Wang J, et al: Kaempferol is an estrogen-related receptor alpha and gamma inverse agonist. FEBS Lett 2009, 583(4):643-7.

39. Hung $\mathrm{H}$ : Inhibition of estrogen receptor alpha expression and function in MCF-7 cells by kaempferol. Journal of Cellular Physiology 2004, 198(2):197-208.

40. Kim HJ, et al: Estrogen receptor alpha-induced cholecystokinin type A receptor expression in the female mouse pituitary. J Endocrinol 2007, 195(3):393-405.

41. Planas-Silva MD, Filatova IS: Estrogen-dependent regulation of Eg5 in breast cancer cells. Anticancer Drugs 2007, 18(7):773-9.

42. Widdop B: Therapeutic drug monitoring. Contemporary issues in clinical biochemistry Edinburgh; New York: Churchill Livingstone; 1985.

43. Willemse PH, et al: Clinical and endocrine effects of cyproterone acetate in postmenopausal patients with advanced breast cancer. Eur J Cancer Clin Oncol 1988, 24(3):417-21.

44. Awasthi N, et al: BMS-754807, a small-molecule inhibitor of insulin-like growth factor-1 receptor/insulin receptor, enhances gemcitabine response in pancreatic cancer. Mol Cancer Ther 2012, 11(12):2644-53.

45. Lee SJ, et al: A pilot study for the early assessment of the effects of BMS754807 plus gefitinib in an $\mathrm{H} 292$ tumor model by [(18)F] fluorothymidine-positron emission tomography. Invest New Drugs 2012.

46. Kolb EA, et al: Initial testing (stage 1) of the IGF-1 receptor inhibitor BMS754807 by the pediatric preclinical testing program. Pediatr Blood Cancer 2011, 56(4):595-603.

47. Carboni JM, et al: BMS-754807, a small molecule inhibitor of insulin-like growth factor-1R/IR. Mol Cancer Ther 2009, 8(12):3341-9.

48. Litzenburger BC, et al: High IGF-IR Activity in Triple-Negative Breast Cancer Cell Lines Correlates with Sensitivity to IGF-IR Inhibitor BMS754807 in This Subtype of Human Breast Cancer. Cancer Research 2009, 69(24):581s-581s.

49. Tamura Y, et al: phase 1 Dose-Escalating Study of Bms-754807 in Japanese Patients with Advanced Solid Tumors. Annals of Oncology 2012, 111-112.

50. Kolb EA, et al: Initial Testing (Stage 1) of the IGF-1 Receptor Inhibitor BMS-754807 by the Pediatric Preclinical Testing Program. Pediatr Blood Cancer 2011, 56(4):595-603.

51. Chu QS, et al: BMS-754807, an oral dual IGF-1R/insulin receptor (IR) inhibitor: initial results from a Phase 1 dose- and schedule-finding study in combination with carboplatin/paclitaxel in subjects with solid tumors. Ejc Supplements 2010, 8(7):131-131.

52. Desai J, et al: Targeting Type I Insulin-Like Growth Factor Receptor and Insulin Receptor for Cancer Therapy: The Oral Dual Inhibitor Bms-754807 in Clinical Development. Annals of Oncology 2010, 9-9.

53. Macdonald RL, Bergey GK: Valproic acid: effect on GABA-mediated postsynaptic inhibition in cultured mammalian spinal cord neurons. Trans Am Neurol Assoc 1978, 254-6.

54. Duenas-Gonzalez A, et al: Valproic acid as epigenetic cancer drug: preclinical, clinical and transcriptional effects on solid tumors. Cancer Treat Rev 2008, 34(3):206-22.

55. Michaelis M, Doerr HW, Cinatl J Jr: Valproic acid as anti-cancer drug. Curr Pharm Des 2007, 13(33):3378-93.

56. Fortunati $\mathrm{N}$, et al: Valproic acid is a selective antiproliferative agent in estrogen-sensitive breast cancer cells. Cancer Lett 2008, 259(2):156-64.

57. Oh DS, et al: Estrogen-regulated genes predict survival in hormone receptor-positive breast cancers. Journal of Clinical Oncology 2006, 24(11):1656-64.

58. Macdonald F: Dictionary of pharmacological agents. London; Weinheim; New York: Chapman \& Hall; 1997, 1 livret d'installation d'un cédérom.

59. Munster PN, et al: Modulation of Hsp90 function by ansamycins sensitizes breast cancer cells to chemotherapy-induced apoptosis in an RB- and schedule-dependent manner. See: E. A. Sausville, Combining cytotoxics and 17-allylamino, 17-demethoxygeldanamycin, sequence and tumor biology matters, Clin. Cancer Res., 7: 2155-2158, 2001. Clin Cancer Res 2001, 7(8):2228-36.

60. Kitson RR, et al: Synthesis of 19-substituted geldanamycins with altered conformations and their binding to heat shock protein Hsp90. Nat Chem 2013, 5(4):307-14.

61. Onuoha SC, et al: Mechanistic studies on Hsp90 inhibition by ansamycin derivatives. J Mol Biol 2007, 372(2):287-97.

62. Bellarosa D, et al: SAHA/Vorinostat induces the expression of the CD137 receptor/ligand system and enhances apoptosis mediated by soluble CD137 receptor in a human breast cancer cell line. Int J Oncol 2012, 41(4):1486-1494.

63. Fiskus $W$, et al: Co-treatment with vorinostat synergistically enhances activity of Aurora kinase inhibitor against human breast cancer cells. Breast Cancer Research and Treatment 2012, 135(2):433-444.

64. Uehara N, Yoshizawa K, Tsubura A: Vorinostat enhances protein stability of p27 and p21 through negative regulation of Skp2 and Cks1 in human breast cancer cells. Oncology Reports 2012, 28(1):105-110.

65. Uehara N, et al: Requirement of p38 MAPK for a cell-death pathway triggered by vorinostat in MDA-MB-231 human breast cancer cells. Cancer Lett 2012, 315(2):112-121.

66. Wong NS, et al: Impact of UDP-gluconoryltransferase 2B17 genotype on vorinostat metabolism and clinical outcomes in Asian women with breast cancer. Pharmacogenet Genomics 2011, 21(11):760-768.

67. Munster PN, et al: A phase II study of the histone deacetylase inhibitor vorinostat combined with tamoxifen for the treatment of patients with hormone therapy-resistant breast cancer. Br J Cancer 2011, 104(12):1828-1835

68. Zhou Q, et al: Screening for therapeutic targets of vorinostat by SILACbased proteomic analysis in human breast cancer cells. Proteomics 2010 10(5):1029-1039.

69. Swaby RF, et al: A Phase II Study of the Histone Deacetylase Inhibitor, Vorinostat, in Combination with Trastuzumab in Patients with Advanced Metastatic and/or Local Chest Wall Recurrent HER-2 Amplified Breast Cancer Resistant to Transtuzumab-Containing Therapy: (E1104) a Trial of the Eastern Cooperative Oncology Group. Cancer Research 2009, 69(24):793s-793s.

70. Palmieri $D$, et al: preclinical studies in support of the use of vorinostat (SAHA) for the treatment of brain metastases of breast cancer. Clinical \& Experimental Metastasis 2009, 26(7):905-906.

71. Munster PN, et al: phase II trial of the histone deacetylase inhibitor, vorinostat, to restore hormone sensitivity to the antiestrogen tamoxifen in patients with advanced breast cancer who progressed on prior hormone therapy. Journal of Clinical Oncology 2009, 27(15).

72. Luu TH, Morgan RJ, Leong L: A Phase II Trial of Vorinostat in Metastatic Breast Cancer (vol 14, pg 7138, 2008). Clinical Cancer Research 2009, 15(1):416-416. 
73. Luu TH, et al: A Phase II Trial of Vorinostat (Suberoylanilide Hydroxamic Acid) in Metastatic Breast Cancer: A California Cancer Consortium Study. Clinical Cancer Research 2008, 14(21):7138-7142.

74. Vanhaecke $T$, et al: Trichostatin A-like hydroxamate histone deacetylase inhibitors as therapeutic agents: toxicological point of view. Current Medicinal Chemistry 2004, 11(12):1629-43.

75. Tavakoli-Yaraki $M$, et al: Induction of apoptosis by Trichostatin A in human breast cancer cell lines: involvement of 15-Lox-1. Tumour Biol 2013, 34(1):241-9.

76. Nakajima S, et al: Trichostatin A with adenovirus-mediated p53 gene transfer synergistically induces apoptosis in breast cancer cell line MDAMB-231. Oncology Reports 2009, 22(1):143-8.

77. Alao JP, et al: Histone deacetylase inhibitor, trichostatin A induces ubiquitin-dependent cyclin D1 degradation in MCF-7 breast cancer cells. Mol Cancer 2006, 8.

78. Min KN, et al: Estrogen receptor enhances the antiproliferative effects of trichostatin A and HC-toxin in human breast cancer cells. Arch Pharm Res 2004, 27(5):554-61.

79. Vigushin DM, et al: Trichostatin A is a histone deacetylase inhibitor with potent antitumor activity against breast cancer in vivo. Clin Cancer Res 2001, 7(4):971-6.

doi:10.1186/1752-0509-7-S5-S5

Cite this article as: Ma et al:: BRCA-Monet: a breast cancer specific drug treatment mode-of-action network for treatment effective prediction using large scale microarray database. BMC Systems Biology 2013 7(Suppl 5):S5

\section{Submit your next manuscript to BioMed Central} and take full advantage of:

- Convenient online submission

- Thorough peer review

- No space constraints or color figure charges

- Immediate publication on acceptance

- Inclusion in PubMed, CAS, Scopus and Google Scholar

- Research which is freely available for redistribution

Submit your manuscript at www.biomedcentral.com/submit 